\title{
DISOLUTION OF STAR CLUSTERS IN GALAXIES
}

\author{
Roland Wielen
}

Astronomisches Rechen-Institut

\section{ABSTRACT}

We present a procedure which allows to predict the dissolution times of star clusters in a simple way. The dissolution time of a cluster depends mainly on its total mass, its median radius and its galactic environment (galactic tidal field and passing massive objects). As an example, we discuss the lifetimes of LMC clusters. Finally, we show that massive black holes, which have been proposed as the major constituent of the dark coronae of galaxies, are very effective in destroying globular clusters.

\section{INTRODUCTION}

Star clusters in galaxies provide of ten more detailed information than individual stars: Clusters are usually brighter, the sample of clusters is relatively more complete, and, most importantly, individual ages of clusters are usually of higher accuracy than those of single stars, and the ages of observable clusters cover the whole range between the youngest and oldest objects in galaxies. Due to internal or external dynamical effects, star clusters often dissolve during a period shorter than the Hubble time. The dynamical dissolution of clusters can therefore strongly determine the distribution of presently observable properties of clusters. It is then more difficult to deduce the distribution of the original properties of clusters at birth. On the other hand, the observable age distribution of open clusters contains the most direct information on the dynamical dissolution of star clusters in a galaxy.

\section{THEORETICAL PREDICTIONS FOR THE LIFETIMES OF STAR CLUSTERS}

There are many mechanisms which are causing the dissolution of star clusters. The most important internal mechanism is the evaporation of stars, due to the exchange of orbital energy during 
close encounters between the cluster members. The nearly stationary tidal field of the parent galaxy can increase the evaporation rate dramatically by lowering the limiting energy for bound stars. Internal evolution of the individual stars causes a mass loss from the cluster, thus weakening the binding of the cluster. Tidal shocks due to passing objects, such as giant molecular clouds, are often a very effective external mechanisms for the partial or complete destruction of clusters.

\subsection{Dissolution Times of Clusters Predicted from N-Body Simulations and Theoretical Extrapolations}

The most direct way to determine theoretically the dissolution times of star clusters are provided by $\mathrm{N}$-body simulations. The results of such N-body simulations have been extensively reviewed

(Aarseth 1985, Aarseth and Lecar 1975, Wielen 1974, 1975, 1985). The most recent $\mathrm{N}$-body experiments have been carried out by $\mathrm{E}$. Terlevich $(1980,1983,1986)$. It is now possible to follow the dynamical evolution of a star cluster essentially until its final dissolution. The main limitation for $\mathrm{N}$-body experiments is set by the initial number $\mathrm{N}$ of stars in a cluster which can be handled on the available computers within reasonable computing times. For star clusters, this limit is presently of the order of $N=1000$ stars. In order to extrapolate the $\mathrm{N}$-body results to higher values of $\mathrm{N}$, we have to rely on theoretical extrapolations.

We consider first the dissolution of a cluster due to the combined effects of the internal relaxation and the stationary tidal field of the galaxy alone. Mass loss of evolving stars is neglected, since it does not seem to alter the results dramatically. Tidal shocks due to passing giant molecular clouds or massive black holes are discussed in Section 2.2 .

\subsubsection{Isolated Clusters}

For isolated clusters, we use the theory developed by Hénon $(1960,1969)$ in order to extrapolate the results of $\mathrm{N}$-body simulations to higher values of $\mathrm{N}$. Hénon's theory is based on the assumption that close encounters between cluster stars are the most important source of escaping stars. This assumption has been nicely confirmed by the $\mathrm{N}$-body simulations, which showed that most of the escapers gain the necessary energy indeed during close encounters with single stars or binaries. This result is not in contradiction to another result of N-body simulations (Aarseth, Hénon and Wielen, 1974), namely that the overall dynamical evolution of a cluster is rather accurately described by a Fokker-Planck equation based on the predominance of more distant, weak encounters. It means only that the rare escape events are not due to the otherwise dominant diffusion of stars in phase space. 
Using Hénon's theory, we predict for the evaporation time $T$,

$$
\mathrm{T}=-\mathrm{N} /(\mathrm{dN} / \mathrm{dt})=\mathrm{NT}_{\mathrm{cr}} / \phi_{0}
$$

The crossing time $T_{c r}$ for a Plummer model is given by

$$
\mathrm{T}_{\mathrm{cr}}=(32 /(3 \pi))^{3 / 2}\left(\mathrm{Gm}_{\mathrm{c}} / \mathrm{r}_{\mathrm{c}}^{3}\right)^{-1 / 2} \text {, }
$$

where $m_{c}$ is the total mass of the cluster, $r_{c}$ is the median radius of the cluster (containing half of $\mathrm{m}_{\mathrm{c}}$ in projection), and $\mathrm{G}$ is the gravitational constant. The quantity $\phi_{0}$ in Eq. 1 is best determined from N-body experiments. For a cluster with a realistic distribution of stellar masses, similar to Salpeter's law, we find for the number of stars which escape during one crossing time:

$$
\phi_{0}=0.56 \quad \text {. }
$$

Eqs. 1, 2 and 3 allow to predict the dissolution time of an isolated star cluster for any initial value of $\mathrm{N}, \mathrm{m}_{\mathrm{c}}$ and $\mathrm{r}_{\mathrm{c}}$, provided that our assumptions are also valid outside the experimentally explored range of $\mathrm{N}$.

\subsubsection{Tidally Limited Clusters}

The theory presented in Section 2.1.1 can be extended to tidally limited clusters. The N-body experiments show that even for tidally limited clusters, close encounters produce still the overwhelming fraction of escapers. It can be shown that, to a first approximation, the number of escapers per crossing time depends on the ratio between the radius $r_{c}$ and the tidal radius $r_{t}$ of the cluster only. This leads to the following prediction for the dissolution time $T$ of tidally limited clusters:

$$
\mathrm{T}=\mathrm{NT} \mathrm{cr}_{\mathrm{r}} /\left(\phi_{0} \mathrm{f}\left(\mathrm{r}_{\mathrm{c}} / \mathrm{r}_{\mathrm{t}}\right)\right)
$$

$\phi_{0}$ is given by Eq. 3, and the function $f\left(r_{c} / r_{t}\right)$ has been derived by fitting the results of $\mathrm{N}$-body simulations of tidally limited clusters (Fig. 1). The fitting curve corresponds to

$$
f\left(r_{c} / r_{t}\right)=\left(1+\left(43 r_{c} / r_{t}\right)^{3}\right)^{1 / 2} \text {. }
$$

This expression has the desired properties: $f$ approaches 1 for small radii $r_{c}$ (isolated clusters), and $T$ becomes independent of $r_{c}$ for large radii $r_{c}$ (strongly tidally limited clusters). The tidal radius $r_{t}$ is given by

$$
r_{t}=\left(G m_{c} /\left(4 \omega^{2}-k^{2}\right)\right)^{1 / 3}
$$

where the local rotational frequency $\omega$ and the local epicyclic frequency $k$ have to be derived from the rotation curve of the parent galaxy. If we introduce a 'tidal time' $T_{t}$, 


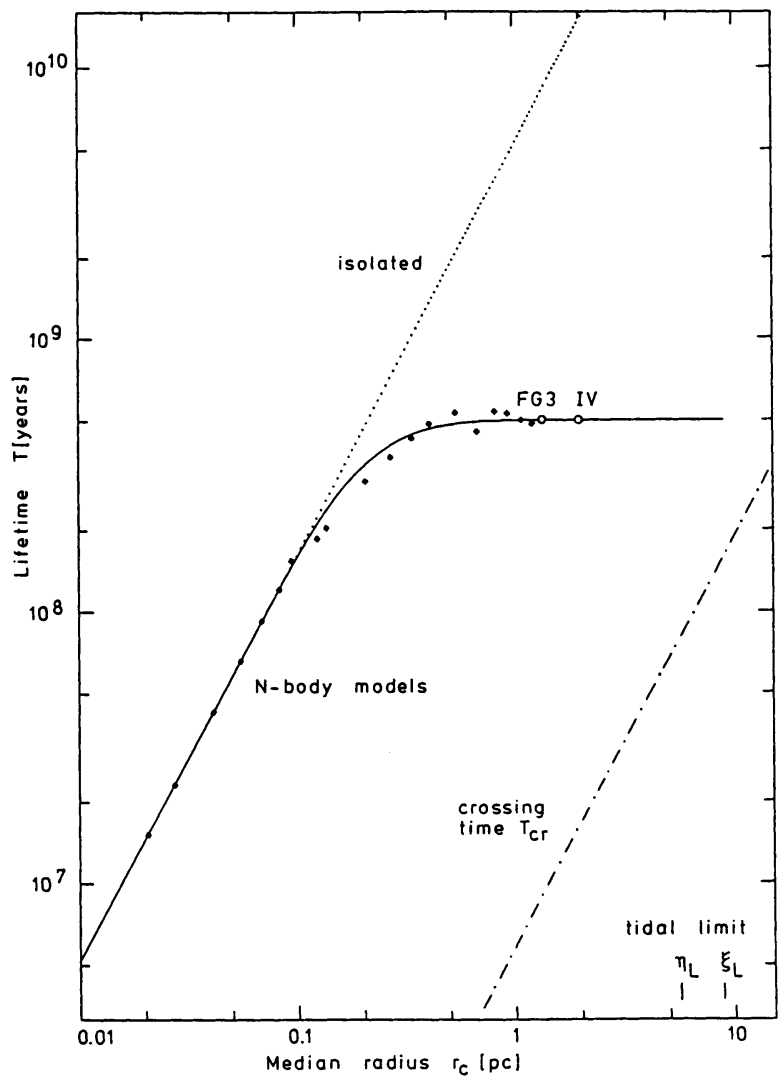

Fig. 1. Fit of the results of $\mathrm{N}$-body simulations for the dissolution time $T$ of a cluster with $N=500$ stars and $m_{c}=250 m_{\odot}$ as a function of the median radius $r_{c}$ of the cluster. N-body models from Wielen (1975, full squares and FG3) and Terlevich (1983, IV). Solid curve: T from Eqs. 4 and 5.

$$
\begin{aligned}
T_{t} & =\left(r_{t} /\left(43 r_{c}\right)\right)^{3 / 2} T_{c r} \\
& =(32 /(3 \pi \cdot 43))^{3 / 2}\left(4 \omega^{2}-\kappa^{2}\right)^{-1 / 2} \\
& =0.022\left(4 \omega^{2}-k^{2}\right)^{-1 / 2},
\end{aligned}
$$

then the function $f$ can be expressed in terms of the ratio between the crossing time $\mathrm{T}_{\mathrm{cr}}$ of the cluster and the tidal time $\mathrm{T}_{\mathrm{t}}$,

$$
f\left(r_{c} / r_{t}\right)=f\left(T_{c r} / T_{t}\right)=\left(1+\left(T_{c r} / T_{t}\right)^{2}\right)^{1 / 2} \text {. }
$$




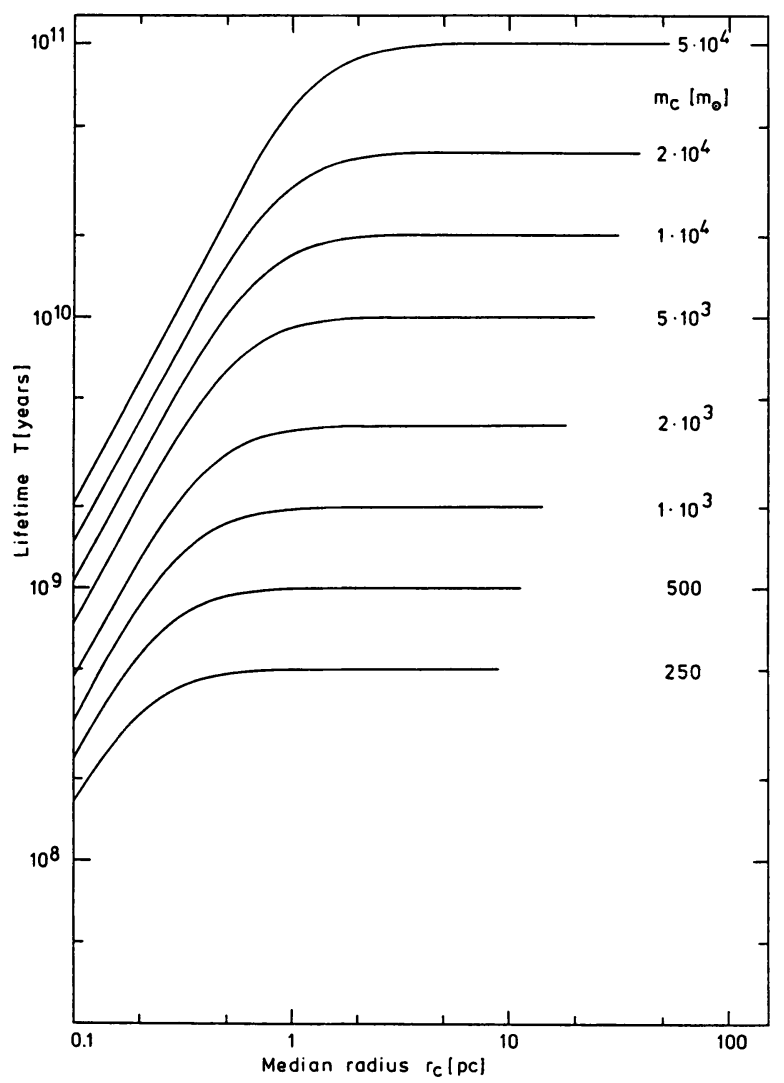

Fig. 2. Dissolution time $T$ of a tidally limited star cluster in the solar neighbourhood as a function of the median radius $r_{c}$ of the cluster for various total masses $m_{c}$, based on Eqs. 4 and 5.

Eqs. 4 and 5 or 8 , together with the expression for $T_{c r}, r_{t}, T_{t}$ and $\phi_{0}$ $=0.56$, provide now the necessary instruments for predicting the dissolution time of a tidally limited star cluster with any value of $\mathrm{N}, \mathrm{m}_{\mathrm{c}}, \mathrm{r}_{\mathrm{c}}$ and $\mathrm{r}_{\mathrm{t}}$.

In Fig. 2, we present results based on our procedure, for star clusters in the solar neighbourhood with a realistic spectrum of stellar masses and a mean stellar mass of $\mathrm{m}_{\mathrm{c}} / \mathrm{N}=0.5 \mathrm{~m}_{\odot}$. The dissolution time $T$ is plotted as a function of $r_{c}$ for various values of $m_{c}$.

In the limit of large cluster radii $r_{c}$, i.e. for strongly tidally limited clusters in which $r_{c}$ is not much smaller than $r_{t}$, we get the following simple expression for the dissolution time: 


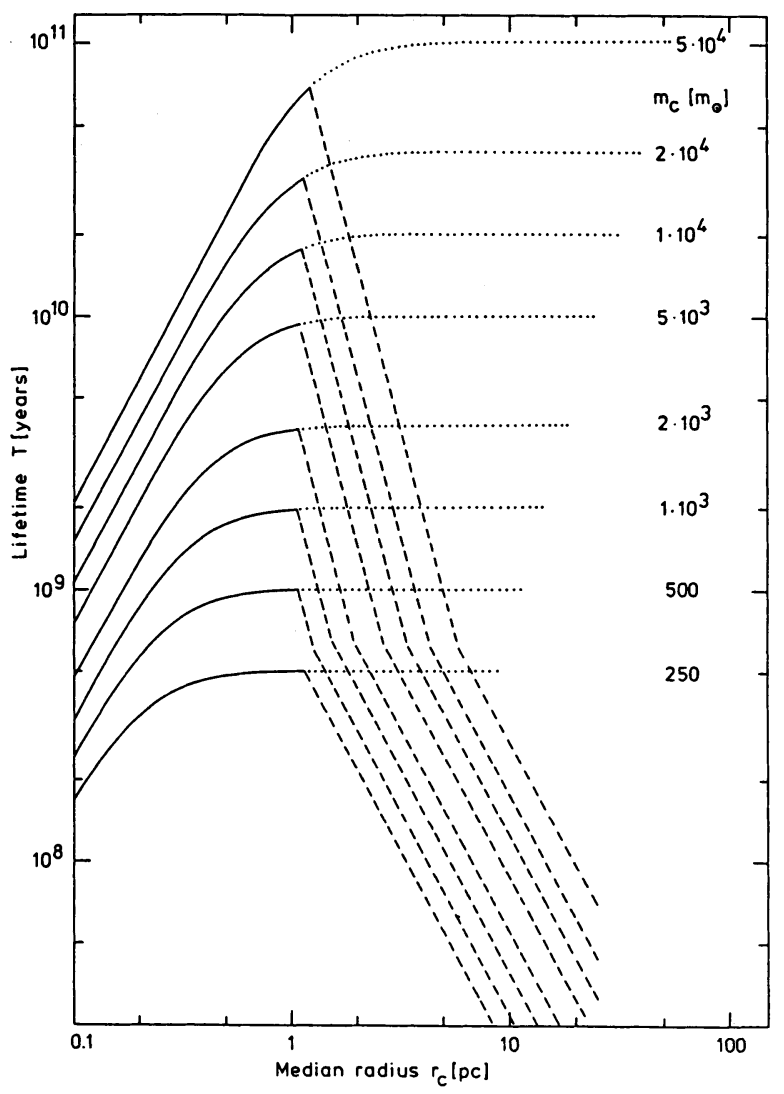

Fig. 3. Dissolution time $T$ of a star cluster in the solar neighbourhood. The full (and dotted) curves are taken from Fig. 2. The dashed lines give the dissolution times due to giant molecular clouds (GMCs).

$$
\mathrm{T}_{\text {lim }}=\mathrm{NT}_{\mathrm{t}} / \phi_{0}
$$

The dissolution time $\mathrm{T}_{1 \mathrm{im}}$ is independent of $\mathrm{r}_{\mathrm{c}}$ and proportional to $\mathrm{N}$, or to $\mathrm{m}_{\mathrm{c}}$ for a fixed value of the mean stellar mass $\left(\mathrm{m}_{\mathrm{c}} / \mathrm{N}\right)$. In the solar neighbourhood, the tidal time $T_{t}$ is about $0.56 \cdot 10^{6}$ years. This leads to $\mathrm{T}_{1 \mathrm{im}}=\mathrm{N} \cdot 10^{6}$ years or, for example, to $\mathrm{T}_{\text {lim }}=10^{10}$ years for $\mathrm{N}=10000$ stars $\left(\mathrm{m}_{\mathrm{c}}=5000 \mathrm{~m}_{\odot}\right.$ for $\left.\mathrm{m}_{\mathrm{c}} / \mathrm{N}=0.5 \mathrm{~m}_{\odot}\right)$. Clusters with $\mathrm{N}$ much higher than $10^{4}$ do not dissolve in a Hubble time, if we consider only internal relaxation and a stationary galactic tidal field. Hence our extrapolation from $N \leq 1000$, covered by $N$-body simulations, to the highest values of real interest, $\mathrm{N} \sim 10^{4}$, is by one order of magnitude only. I would consider that as not too risky. 


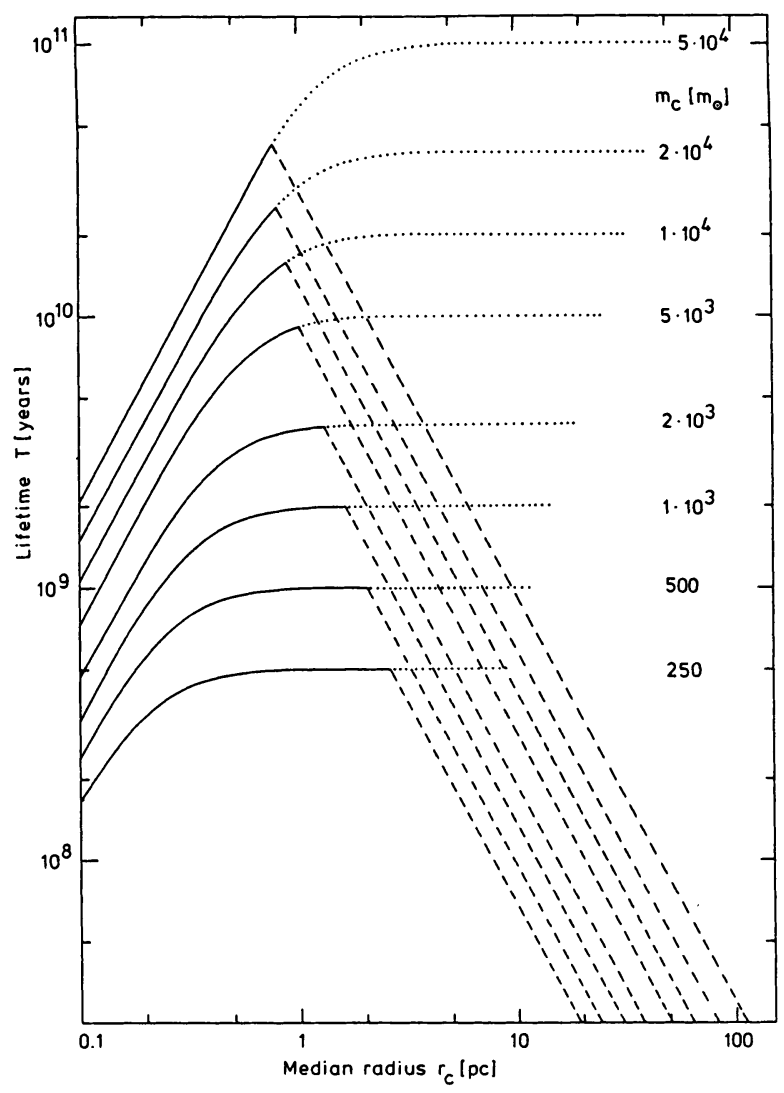

Fig. 4. Dissolution time $\mathrm{T}$ of a star cluster in the solar neighbourhood. The full (and dotted) curves are taken from Fig. 2. The dashed lines give the dissolution times due to massive black holes (MBHs).

\subsection{Dissolution Times of Clusters due to Passing Massive Objects}

Star clusters can also be dissolved by passing massive objects. Important effects can be caused especially by giant molecular clouds (GMC) or by massive black holes (MBH). For a detailed discussion we refer to an earlier paper (Wielen, 1985). For completeness we repeat here the final results for the dissolution times $\mathrm{T}_{\mathrm{n}}$ of star clusters due to passing massive objects. A massive object is characterized by its mass $m_{n}$ and its radius $r_{n} ; \nu_{n}$ is the number density of the massive objects in the neighbourhood of the cluster; $\rho_{a n}=m_{n} \nu_{n}$ is the overall mass density of the objects; $\mathrm{V}$ is the relative velocity between the object and the cluster; and $\alpha r_{c}$ is the effective radius of the cluster 
for tidal shocks ( $\alpha=1$ may be used as a first guess).

Two different cases for the dissolution of clusters by passing objects occur. They are characterized by the relative size of the limiting impact parameter po,

$$
\mathrm{p}_{0}=\max \left(r_{n}, r_{c}\right) \text {, }
$$

with respect to the critical impact parameter $\mathrm{p}_{1}$,

$$
p_{1}=4(\alpha / 3)^{1 / 2}(G / \pi)^{1 / 4} m_{n}^{1 / 2} v^{-1 / 2}\left(m_{c} / r_{c}^{3}\right)^{-1 / 4} \text {. }
$$

In the case of $p_{1}>p_{0}$, the cluster can be destroyed completely by a single encounter, if the massive object passes with an impact parameter $p<p_{1}$. In this case $\left(p_{1}>p_{0}\right)$, the mean dissolution time $\mathrm{T}_{\mathrm{n}, 1}$ of a cluster is given by

$$
\mathrm{T}_{\mathrm{n}, 1}=(3 /(32 \alpha)) \pi^{-1 / 2}\left(\mathrm{Gm}_{\mathrm{c}} / \mathrm{r}_{\mathrm{c}}^{3}\right)^{1 / 2} /\left(\mathrm{G} \rho_{\mathrm{an}}\right) \text {. }
$$

It is remarkable that $T_{n}, 1$ depends only on the overall mass density $\rho_{a n}$, but not on the individual values of $m_{c}, r_{c}$ or $V$ of the passing objects.

In the case $p_{0}>p_{1}$, a number of successive encounters are necessary for disrupting the cluster. The resulting dissolution time $\mathrm{T}_{\mathrm{n}, 0}$ of a cluster is given by

$$
T_{n, 0}=\left(9 /\left(512 \alpha^{2}\right)\right) G^{-1} m_{n}^{-1} \rho_{a n}^{-1} V_{c} m_{c}{ }_{c}^{-3} p_{0}^{2},
$$

where $p_{0}$ is either $r_{n}$ or $r_{c}$ according to Eq. 10 .

Both expressions, $T_{n, 0}$ and $T_{n, 1}$, are only valid if the encounter between the cluster and the object is impulsive (Spitzer, 1958), i.e. if $\mathrm{p} / \mathrm{V}<\mathrm{T}_{\mathrm{cr}}$. This is essentially fulfilled if

$$
\max \left(\mathrm{p}_{0}, \mathrm{p}_{1}\right)<\mathrm{V} \mathrm{T}_{\mathrm{cr}} \quad \text {. }
$$

In order to calculate $T_{n}$ for a given cluster, one has firstly to determine $p_{0}$ and $p_{1}$ from Eqs. 10 and 11, secondly to check the impulsive approximation according to Eq. 14, and then finally to compute $T_{n}$ either from Eq. $12\left(T_{n, 1}\right.$ for $\left.p_{1}>p_{0}\right)$ or from Eq. $13\left(T_{n, 0}\right.$ for $\left.p_{0}>p_{1}\right)$. The resulting dissolution time $T_{n}$ neglects the effect of the galactic tidal field, which would tend to reduce the values for $T_{n}$, because it again lowers the limiting energy for bound stars.

In Figs. 3 and 4 , we have plotted the dissolution time $T_{n}$ of clusters as dashed lines, either due to giant molecular clouds (Fig. 3) or massive black holes (Fig. 4). The parameters used are $m_{n}=5 \cdot 105$ $\mathrm{m}_{\odot}, \mathrm{r}_{\mathrm{n}}=25 \mathrm{pc}, \rho_{\mathrm{an}}=0.02 \mathrm{~m}_{\odot} / \mathrm{pc}^{3}$ and $\mathrm{V}=10 \mathrm{~km} / \mathrm{s}$ for GMCs, and $\mathrm{m}_{\mathrm{n}}=$ $3 \cdot 10^{6} \mathrm{~m}_{\odot}, r_{\mathrm{n}}=0, \rho_{\mathrm{an}}=0.006 \mathrm{~m} / \mathrm{pc}^{3}$ and $\mathrm{V}=250 \mathrm{~km} / \mathrm{s}$ for $\mathrm{MBHs}$ (see Wielen, 1985). 


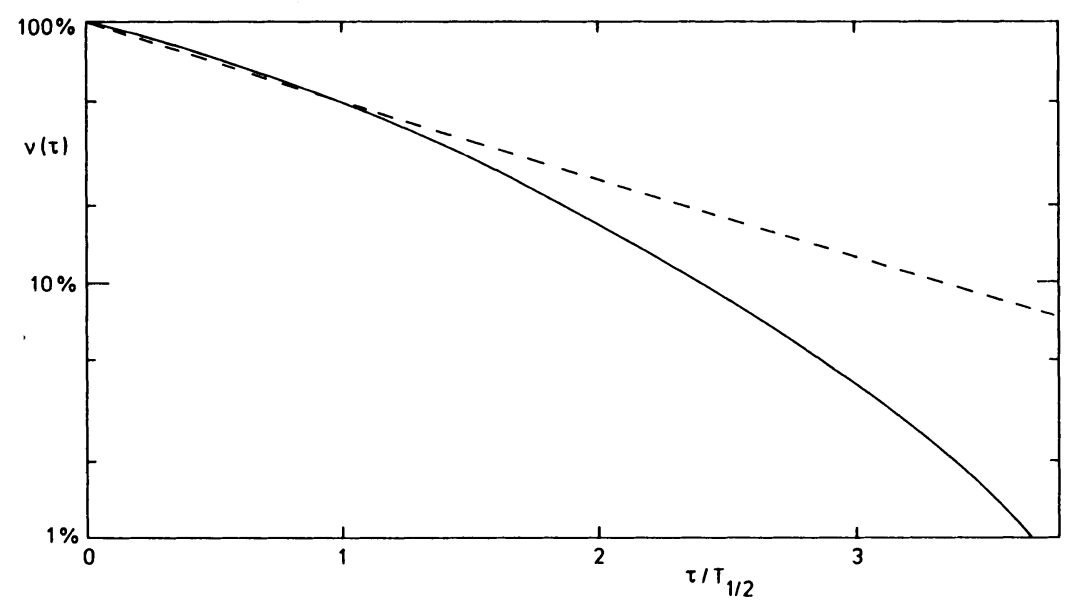

Fig. 5. Survival rate $\nu$ of clusters with the same initial values of $\mathrm{N}, \mathrm{m}_{\mathrm{c}}$ and $\mathrm{r}_{\mathrm{c}}$ as a function of the age $\tau$ (scaled by the median value $\mathrm{T}_{1 / 2}$ of the dissolution times). Disruption of clusters due to GMCs (or MBHs) in a case with $\mathrm{p}_{1} \gg \mathrm{p}_{0}$. Evaporation due to internal relaxation neglected here.

If a type of clusters may be destroyed completely by a single encounter with a passing object, i.e. if $\mathrm{p}_{1}>\mathrm{p}_{0}$, then there is a wide spread in the actual lifetimes of individual clusters, caused by the stochastic occurence of such encounters. Hence the dissolution time $T_{n}$ derived above has to be interpreted as an expectation value (mean value) for the lifetime only. Kuhrau and Wielen (unpublished) have carried out Monte-Carlo simulations on the disruption of clusters due to passing objects. A typical result of these studies is shown in Fig. 5. The full curve gives the survival rate $\nu(\tau)$, i.e. the percentage of clusters which live longer than $\tau$, for a case with $p_{1} \gg p_{0}$. All clusters had initially the same total mass and radius. The initial decline of $\nu(\tau)$ is roughly exponential (dashed line), as expected for random close encounters. For larger ages $\tau$, the accumulating number of distant encounters causes a steeper decline of $\nu$.

\subsection{Total Dissolution Times}

In order to derive the dissolution time $T_{\text {total }}$, due to the combined effect of internal relaxation and passing objects, we add the corresponding escape rates, $1 / \mathrm{T}$ :

$$
\mathrm{T}_{\text {total }}^{-1}=\mathrm{T}_{\mathrm{N}-\text { body }}^{-1}+\mathrm{T}_{\mathrm{GMC}}^{-1}+\mathrm{T}_{\mathrm{MBH}}^{-1} \text {. }
$$

Here, $\mathrm{T}_{\mathrm{N} \text {-body }}$ is to be taken from Section 2.1, while $\mathrm{T}_{\mathrm{GMC}}$ and $\mathrm{T}_{\mathrm{MBH}}$ have to be derived from Section 2.3. In Figs. 3 and 4, we have not 


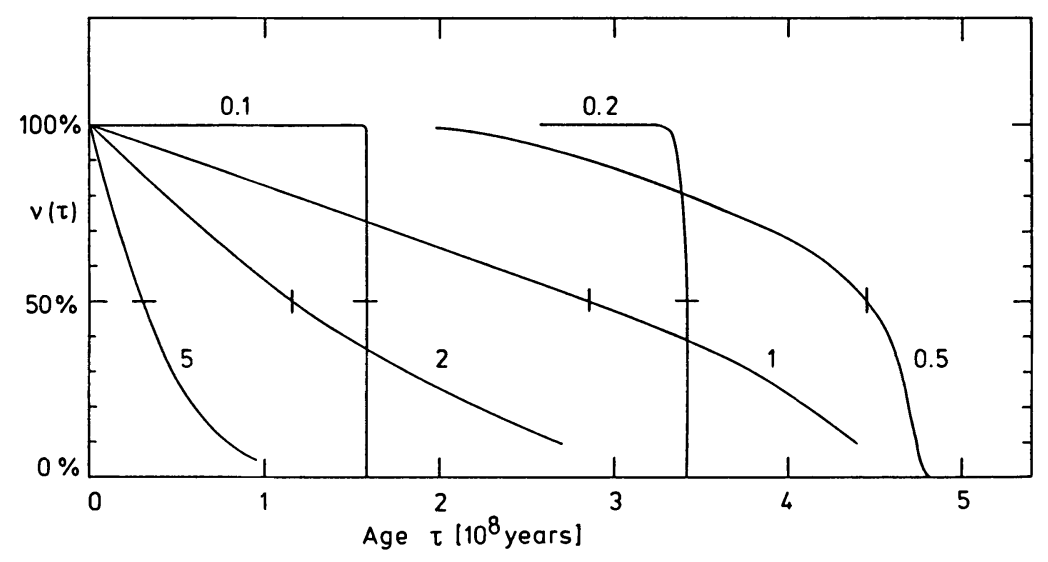

Fig. 6. Survival rate $\nu$ of clusters with the same initial values of $\mathrm{m}_{\mathrm{C}}=250 \mathrm{~m}_{\odot}, \mathrm{N}=500$ stars and various values of $r_{c}$ (in pc) as a function of age $\tau$. Dissolution of clusters due to internal relaxation, galactic tidal field and GMCs.

plotted the total dissolution time, since this would have obscured the picture. An example of a curve for the total dissolution time is shown in Fig. 1 of Wielen (1985). At the 'crossing points', the total dissolution time is half of $\mathrm{T}_{\mathrm{N} \text {-body }}=\mathrm{T}_{\mathrm{GMC}}$, while for small (large) radii, $\mathrm{T}_{\text {total }}$ approaches $\mathrm{T}_{\mathrm{N} \text {-body }}\left(\mathrm{T}_{\mathrm{GMC}}\right)$.

As discussed in Section 2.2, close encounters with passing massive objects produce a wide distribution of actual lifetimes, even for clusters with the same initial values of $m_{c}, r_{c}$ and $r_{t}$. To the contrary, the internal relaxation leads to rather well-defined dissolution times. The width of the distribution function of total lifetimes depends therefore on the relative importance of internal relaxation and close encounters with GMCs or MBHs. An example for the survival rate $\nu(\tau)$ of clusters with $m_{c}=250 \mathrm{~m}_{\odot}, N=500$ stars and various values of $r_{c}$ is shown in Fig. 6 (Kuhrau and Wielen, unpublished).

\section{STAR CLUSTERS IN THE LARGE MAGELLANIC CLOUd (LMC)}

We will now apply the procedure presented in Section 2 to star clusters in the LMC. For deriving the tidal time $\mathrm{T}_{t}$ (Eq.7) as a function of the distance $R$ from the center of the LMC, we need the run of $4 \omega^{2}-K^{2}$ based on the rotation curve of the LMC. We use the results of Elson and Freemann (1986). The tidal time in the LMC increases from $0.4 \cdot 10^{6}$ years at $R \sim 10$ to $1.1 .10^{6}$ years at $R \sim 6^{\circ}$. At $R \sim 3^{\circ}$, the tidal time in the LMC is just about equal to the value in the solar neighbourhood, $0.6 \cdot 10^{6}$ years, so that $\mathrm{Fig} .2$ can be directly used for LMC clusters at $R \sim 3^{\circ}$. 
If we consider only the dissolution of clusters due to internal relaxation combined with the LMC tidal field, then all clusters with median radii $r_{c}$ larger than about $1 \mathrm{pc}$ and with total masses $\mathrm{m}_{\mathrm{c}}$ above $7500 \mathrm{~m}_{\odot}$ at $\mathrm{R} \sim 3^{\circ}$, above $9000 \mathrm{~m}_{\odot}$ at $\mathrm{R} \sim 1^{\circ}$, and above $4000 \mathrm{~m}_{\odot}$ at $R \sim 6^{\circ}$ should have survived over $15 \cdot 10^{9}$ years. Clusters with smaller total masses would live shorter, in direct proportion to their value of $m_{c}$.

The overall density of molecular gas in the LMC is still rather uncertain (Israel, 1984, Cohen et al., 1984). Hence it is essentially impossible to estimate at present the effect of molecular clouds on LMC clusters. Since the molecular gas seems to be less abundant in the LMC than in our Galaxy, its effect on LMC clusters is correspondingly smaller than for galactic clusters. For LMC clusters with large median radii, however, large molecular clouds may still be the dominant source of cluster dissolution.

Elson and Fall (1985) have derived the age distribution for rich star clusters in the LMC from observational data. They find a decrease of the number of clusters with increasing age similar to that of open clusters in our Galaxy (Wielen, 1971) for ages up to $3 \cdot 10^{8}$ years, and a flatter tail for older LMC clusters. The indicated short median dissolution time of about $2 \cdot 10^{8}$ years for these rich LMC clusters with $\mathrm{m}_{\mathrm{c}}>10^{3} \mathrm{~m}_{\odot}$ would require strong effects from passing massive objects such as giant molecular clouds. However, due to the various assumptions which enter into the derivation of the 'observed' age distribution of LMC clusters, this conclusion is quite uncertain at present. Mateo (1986) has presented an age distribution for less massive LMC clusters which differs strongly from the results of Elson and Fall. Mateo's data indicate a median dissolution time of about $3 \cdot 10^{9}$ years for a constant formation rate. The discrepancy may be, at least partially, due to the different locations of the cluster samples (typically $R \sim 2^{\circ}$ for Elson and Fall, and $R \sim 6^{\circ}$ for Mateo). Mateo's results can be explained, if the effect of GMCs are negligible in the LMC at $R \sim 6^{\circ}$. For this distance, we would then predict a dissolution time of $2 \cdot 10^{9}$ years for a cluster with $\mathrm{m}_{\mathrm{c}}=500 \mathrm{~m}_{\odot}$ and $r_{\mathrm{c}}>1 \mathrm{pc}$.

\section{THE EFFECT OF MASSIVE BLACK HOLES ON GLOBULAR CLUSTERS}

Massive black holes (MBHs) have been proposed by Lacey and Ostriker (1985) and Ipser and Semenzato (1985) as the major constituents of dark galactic coronae. These MBHs would nicely explain the increase in the velocity dispersion of disk stars as a function of age as derived by Wielen (1977). If the MBHs really exist, they would be extremely efficient in dissolving globular clusters.

In Table 1, we give the dissolution times for globular clusters of various total masses and median radii, at the solar distance from the galactic center, due to MBHs. For the MBHs, we use the same 
Table I

Disruption time $\mathrm{T}_{\mathrm{n}}$ (in 109 years) for

globular clusters due to massive black holes

\begin{tabular}{|c|c|c|c|c|c|}
\hline $\begin{array}{l}\text { mass } \mathrm{m}_{\mathrm{c}} \\
\left(\text { in } \mathrm{m}_{\odot}\right) \\
\text { of the } \\
\text { cluster }\end{array}$ & $\begin{array}{c}\text { media } \\
1\end{array}$ & $\begin{array}{c}\text { dius } x \\
2\end{array}$ & $\begin{array}{l}\text { (in } p c \\
5\end{array}$ & of the & uster \\
\hline $2 \cdot 10^{6}$ & 188 & 69 & 28 & 14 & 6.9 \\
\hline $1 \cdot 10^{6}$ & 133 & 47 & 14 & 6.9 & 3.5 \\
\hline $5 \cdot 10^{5}$ & 94 & 33 & 8.4 & 3.5 & 1.7 \\
\hline $2 \cdot 10^{5}$ & 59 & 21 & 5.3 & 1.9 & 0.7 \\
\hline $1 \cdot 105$ & 42 & 15 & 3.8 & 1.3 & 0.5 \\
\hline $5 \cdot 10^{4}$ & 30 & 11 & 2.7 & 0.9 & 0.3 \\
\hline $2 \cdot 10^{4}$ & 19 & 6.6 & 1.7 & 0.6 & 0.2 \\
\hline $1 \cdot 10^{4}$ & 13 & 4.7 & 1.2 & 0.4 & 0.1 \\
\hline
\end{tabular}

Table II

Survival rate $\nu$ (in $\%$ ) for globular clusters of age $\tau=15 \cdot 10^{9}$ years in the presence of massive black holes

\begin{tabular}{|c|c|c|c|c|c|}
\hline $\begin{array}{l}\text { mass } \mathrm{m}_{\mathrm{c}} \\
\left(\text { in } \mathrm{m}_{\odot}\right) \\
\text { of the } \\
\text { cluster }\end{array}$ & $\begin{array}{c}\text { med } \\
1\end{array}$ & radi & $\begin{array}{c}r_{c}(i n \\
5\end{array}$ & $\begin{array}{l}\text { c) of } t h \\
10\end{array}$ & $\begin{array}{c}\text { cluster } \\
20\end{array}$ \\
\hline $2 \cdot 10^{6}$ & 96 & 89 & $(71)$ & $((47))$ & $((19))$ \\
\hline $1 \cdot 10^{6}$ & 94 & 83 & 47 & (19) & $((1))$ \\
\hline $5 \cdot 10^{5}$ & 92 & 75 & 24 & 1 & 0 \\
\hline $2 \cdot 10^{5}$ & 87 & 62 & 8 & 0 & 0 \\
\hline $1 \cdot 105$ & 80 & 50 & 1 & 0 & 0 \\
\hline $5 \cdot 10^{4}$ & 73 & 36 & 0 & 0 & 0 \\
\hline $2 \cdot 10^{4}$ & 59 & 15 & 0 & 0 & 0 \\
\hline $1 \cdot 10^{4}$ & 44 & 5 & 0 & 0 & 0 \\
\hline
\end{tabular}


parameters as in Section 2.2, namely $\mathrm{m}_{\mathrm{n}}=3 \cdot 10^{6} \mathrm{~m}_{\odot}$ and $\rho_{\text {an }}=0.006 \mathrm{~m}_{\odot} / \mathrm{pc}^{3}$. For the relative velocity between MBHs and globular clusters, we assume $\mathrm{V}=2^{1 / 2} \cdot 220 \mathrm{~km} / \mathrm{s}=311 \mathrm{~km} / \mathrm{s}$. A typical globular cluster with $\mathrm{m}_{\mathrm{c}}=2 \cdot 10^{5} \mathrm{~m}_{\odot}$ and $\mathrm{r}_{\mathrm{c}}=5 \mathrm{pc}$ has a mean lifetime $\mathrm{T}_{\mathrm{n}}$ of only $5 \cdot 10^{9}$ years, i.e. about one third of the Hubble time. Clusters with larger radii or smaller masses are even more vulnerable by MBHs.

The dissolution times of globular clusters are inversely proportional to the overall mass density $\rho_{\text {an }}$ of the MBHs in the corona. Therefore, the dissolution time $\mathrm{T}_{\mathrm{n}}$ of a globular cluster depends strongly on its (mean) galactocentric distance $R, T_{n}$ being shorter in the inner parts of the Galaxy and longer in the outer regions. The decrease of $\rho_{a n}$ at larger distances follows the relation $\rho_{a n} \propto R^{-2}$ for a flat rotation curve. This would imply $T_{n} \propto R^{2}$. The increase of $\rho_{a n}$ towards the galactic center is not well-known quantitatively. According to Lacey and Ostriker (1985), the central density $\rho_{a n}$ of MBHs may be higher than their local density by a factor of 2 or more. The dissolution times of globular clusters due to MBHs in the central region of the Galaxy are then correspondingly shorter than the values given in Table $I$.

It may seem at first that dissolution times significantly shorter than the Hubble time would be a strong argument against the existence of MBHs, since the observed globular clusters have ages of about $15 \cdot 10^{9}$ years. One should remember, however, that the disruption of globular clusters by MBHs is a highly stochastic process, leading to a wide spread in the lifetimes of clusters (Section 2.2). We have therefore derived the percentage $\nu$ of globular clusters which survive over a period of $15 \cdot 10^{9}$ years as a function of $m_{c}$ and $r_{c}$. The survival rates $\nu$ presented in Table 2 are based on the dissolution times given in Table 1 , and on a relative survival function similar to that shown in Fig. 5. From Table 2, we see, for example, that $8 \%$ of globular clusters with an initial total mass of $\mathrm{m}_{\mathrm{c}}=2 \cdot 10^{5} \mathrm{~m}_{\odot}$ and a median radius of $r_{c}=5$ pc would survive over $15 \cdot 10^{9}$ years at the solar distance $R_{O}$. This means that in order to explain the presently observed number of such clusters, the initial population of clusters of that type had to be larger than now by a factor of $1 / 0.08=12.5$. The general conclusion is that if the proposed massive black holes do exist, the initial number of globular clusters was much higher than now. It may even be that all halo field stars were formerly members of now dissolved clusters.

A possible argument against the existence of MBHs would come from a universal distribution function for the total luminosities of globular clusters. It is clear from Tables I and II that MBHs would strongly modify any initial distribution of total masses of globular clusters and would probably even determine the location of the peak in the luminosity function of globular clusters and the shape of this function for low cluster luminosities. In galaxies like our one, the dependence of $T_{n}$ on $R$ would produce a strong radial change in the 
luminosity function, which does not seem to be observed. From galaxy to galaxy, one would expect a variation in the location of the maximum of the luminosity function of globular clusters, if there is not a kind of universal coupling between the distribution of globular clusters and the density distribution of MBHs in dark coronae of galaxies.

\section{REFERENCES}

Aarseth, S. J. 1985 in Multiple Time Scales (Computational

Techniques), J. U. Brackbill and B. I. Cohen, eds., Academic Press, Orlando, p. XXX.

Aarseth, S. J. and Lecar, M. 1975 Ann. Rev. Astron. Astrophys. 13,1 .

Aarseth, S. J., Henon, M. and Wielen, R. 1974 Astron. Astrophys. $37,183$.

Caldwe11, J. A. R. and Ostriker, J. P. 1981 Astrophys. J. 251, 61. Cohen, R., Montani, J. and Rubio, M. 1984 in IAU Symposium No. 108. Structure and Evolution of the Magellanic Clouds S. van den Bergh and K. S. de Boer, eds., D. Reidel Publ. Co., Dordrecht, p. 401. Elson, R. A. W. and Fal1, S. M. 1985 Astrophys. J. 299, 211. E1son, R. A. W. and Freeman, K. C. 1986 preprint.

Henon, M. 1960 Ann. Astrophys. 23, 668.

Henon, M. 1969 Astron. Astrophys. 2, 151.

Ipser, J. R. and Semenzato, R. 1985 Astron. Astrophys. 149, 408.

Israe1, F. P. 1984 in IAU Symposium No. 108, Structure and Evolution of the Magellanic Clouds S. van den Bergh and K. S. de Boer, eds., D. Reidel Pub1. Co., Dordrecht, p. 319.

Lacey, C. G. and Ostriker, J. P. 1985 Astrophys. J. 299, 633.

Mateo, M. 1987 in IAU Symposium No. 126. Globular Cluster Systems in Galaxies, J. E. Grindlay and A. G. D. Philip, eds., Reidel, Dordrecht, p. 557.

Spitzer, L. 1958 Astrophys. J. 127, 17.

Terlevich, E. 1980 in IAU Symposium No. 85. Star Clusters, J. E. Hesser, ed., D. Reidel Publ. Co., Dordrecht, p. 165.

Terlevich, E. 1983 Ph.D. Dissertation, Univ. Cambridge, England. Terlevich, E. 1986 Preprint.

Wielen, R. 1971 Astron. Astrophys. 13, 309.

Wielen, R. 1974 in Proceedings of the First European Astronomical

Meeting, Vo1. 2, Stars and the Milky Way System L. N. Mavridis, ed., Springer-Verlag, Berlin, p. 326.

Wielen, R. 1975 in IAU Symposium No. 69. Dynamics of Stellar Systems A. Hayli, ed., D. Reidel Publ. Co., Dordrecht, p. 119.

Wielen, R. 1977 Astron. Astrophys. 60, 263.

Wielen, R. 1985 in IAU Symposium No. 113. Dynamics of Star Clusters

J. Goodman and P. Hut, eds., D. Reidel Publ. Co., Dordrecht,

p. 449 .

Note added in proof: Elson and Freeman 1986 should be replaced by Elson, R. A. W., Fall, S. M. and Freeman, K. C. 1987, preprint. 


\section{DISCUSSION}

INAGAKI: Does the disruption time become significantly shorter if you take into account the post-collapse expansion?

WIELEN: The N-body simulations of cluster dissolution cover in many cases the 'post-collapse' phase. There is no indication for a significant change of the escape rate with time. In the case of an encounter between a cluster and a passing massive object, the actual radius of the cluster at that time determines the fate of the cluster. This effective radius of the cluster may be, of course, affected by post-collapse expansion.

SCHOMMER: Two comments on your LMC comparison. It would be relatively easy for someone to measure tidal radii, using CCD frames from small telescopes, of many LMC clusters. Secondly, while I find the Elson and Fall analysis impressive, I am worried about their sample completeness. In particular, they need to correct the Van den Bergh compilation by counting from Hodge Atlas Plates, by factors of 4-5 for the faintest, oldest clusters, precisely those with $t>10^{9} \mathrm{yrs}$, which lie away from your galactic line; these correction factors may vary from region to region, which could easily bring their age distribution up to that of Mateo.

COHEN: What effect do those proposed massive black holes have on the velocity dispersion of halo stars?

WIELEN: The relative increase in the velocity dispersion of halo stars, due to the proposed massive black holes, is rather small at the solar distance. To get a first guess, one may just add quadratically the velocity dispersion of the oldest disk stars $(\sim 80 \mathrm{~km} / \mathrm{s})$ to the total velocity dispersion of halo stars (say $270 \mathrm{~km} / \mathrm{s}$ ). This leads to about $280 \mathrm{~km} / \mathrm{s}$, or an increase by 48 . In the inner part of the Galaxy, the increase in the velocity dispersion of halo stars by black holes is larger, but probably still not overwhelming, depending mainly on the assumed increase in the number density of the black holes towards the galactic center.

BHATIA: What is the effect of the globular/open clusters on the molecular clouds?

WIELEN: The passing clusters will increase the internal energy of the molecular clouds by tidal heating. Since most open clusters have much smaller masses than giant molecular clouds, the effect is probably small in most cases.

INNANEN: There is a relative absence of globular clusters between $R$ $20 \mathrm{kpc}$ and $\mathrm{R}-70 \mathrm{kpc}$. Could the proposed black holes selectively destroy clusters that once might have been there? 
WIELEN: Probably not. The disruption time for a given type of globular clusters is mainly proportional to the overall density of the black holes. This density is probably decreasing monotonically with increasing galactocentric distance $R$. There is also no indication that the initial masses or radii of globular clusters vary in a dramatical way with $R$.

KING: Can you estimate how much your results would change if it turned out that in the richer clusters the dominant escapes are due to Fokker-Planck diffusion?

WIELEN: In the case of a Fokker-Planck diffusion, the evaporation time of a cluster would increase only as $N / \log _{10}(0.4 \mathrm{~N})$ instead of $\mathrm{N}$. For example, extrapolating from $N=500$ to 5000 stars, the resulting evaporation time for $N=5000$ would have to be decreased by a factor of $\log (0.4 \times 500) / \log (0.4 \times 5000)=0.7$ relative to my results based on escapers by close encounters. Even for a globular cluster with $\mathrm{N}=10^{6}$ stars, the reduction factor would be only about 0.4 . 